\title{
Cohomological characterisation of Steiner bundles
}

\author{
Rosa Maria Miró-Roig and Helena Soares \\ (Communicated by Ronald Fintushel)
}

\begin{abstract}
A vector bundle $E$ on a smooth irreducible algebraic variety $X$ is called a Steiner bundle of type $\left(F_{0}, F_{1}\right)$ if it is defined by an exact sequence of the form

$$
0 \rightarrow F_{0}^{S} \stackrel{\varphi}{\rightarrow} F_{1}^{t} \rightarrow E \rightarrow 0
$$

where $s, t \geq 1$ and $\left(F_{0}, F_{1}\right)$ is a strongly exceptional pair of vector bundles on $X$ such that $F_{0}^{\vee} \otimes F_{1}$ is generated by global sections.

Let $X$ be a smooth irreducible projective variety of dimension $n$ with an $n$-block collection $\mathscr{B}=$ $\left(\mathscr{E}_{0}, \mathscr{E}_{1}, \ldots, \mathscr{E}_{n}\right), \mathscr{E}_{i}=\left(E_{1}^{i}, \ldots, E_{\alpha_{i}}^{i}\right)$, of locally free sheaves on $X$ which generate $D^{b}\left(\mathscr{O}_{X}-\bmod \right)$. We give a cohomological characterisation of Steiner bundles of type $\left(E_{i_{0}}^{a}, E_{j_{0}}^{b}\right)$ on $X$, where $0 \leq a<b \leq n$ and $1 \leq i_{0} \leq \alpha_{a}, 1 \leq j_{0} \leq \alpha_{b}$.
\end{abstract}

2000 Mathematics Subject Classification: 14F05, 14J10, $14 J 60$.

\section{Introduction}

Since Horrocks' criterion [23], which states that a vector bundle on a projective space decomposes into a direct sum of line bundles if and only if it has no intermediate cohomology, cohomological characterisation has become a useful tool to study vector bundles. Several authors, such as Ottaviani [33, 35], Madonna and Chiantini [29, 30, 31, 12, 11], Arrondo, Costa and Graña [2, 3], Faenzi [18, 19], or Costa and Miró-Roig [13], have obtained cohomological classifications of vector bundles on varieties other than the projective spaces, approaching either the problem of whether they split or whether they have no intermediate cohomology. Cohomological characterisation was also used by Horrocks in [24] as a tool to characterise the sheaf of $p$-differential forms on $\mathbb{P}^{n}$. This result was later generalised in [13] to vector bundles on multiprojective spaces.

The first author was partially supported by MTM2007-67704.

The second author was partially supported by Fundação para a Ciência e Tecnologia under grant SFRH/BD/16589/2004, and by Departamento de Métodos Quantitativos do Instituto Superior de Ciências do Trabalho e da Empresa. 
Our goal is to characterise cohomologically Steiner bundles on smooth projective varieties. Steiner bundles were first defined by Dolgachev and Kapranov in [16] as vector bundles $E$ on a projective space $\mathbb{P}^{n}$ defined by an exact sequence of the form

$$
0 \rightarrow \mathrm{O}_{\mathbb{P}^{n}}(-1)^{s} \rightarrow \mathrm{O}_{\mathbb{P}^{n}}^{t} \rightarrow E \rightarrow 0 .
$$

These bundles had been considered earlier by others (see [17], [5]) and, after [16], they have been studied by many authors such as Vallès [38, 39], Ancona and Ottaviani [1], Cascini [9], Brambilla [7, 6], Jardim and Martins [25] and also by us. More precisely, in [36] we defined and studied Steiner bundles on the smooth hyperquadric $Q_{n} \subset \mathbb{P}^{n+1}$.

So far a few cohomological characterisations of Steiner bundles have been given. In [16] Dolgachev and Kapranov showed that a vector bundle $E$ on $\mathbb{P}^{n}$ is a Steiner bundle defined by an exact sequence of type

$$
0 \rightarrow \mathrm{O}_{\mathbb{P}^{n}}(-1)^{s} \rightarrow \mathrm{O}_{\mathbb{P}^{n}}^{t} \rightarrow E \rightarrow 0
$$

if and only if $H^{q}\left(E \otimes \Omega_{\mathbb{P}^{n}}^{p}(p)\right)=0$ for $q>0$ and also for $q=0, p>1$. More recently, Jardim and Martins [25] gave an alternative cohomological characterisation of Steiner bundles on $\mathbb{P}^{n}$ defined as above and moreover, they gave a cohomological characterisation of Steiner bundles on the smooth hyperquadric $Q_{n} \subset \mathbb{P}^{n+1}, n \geq 3$, defined by

$$
0 \rightarrow \mathbb{O}_{Q_{n}}(-1)^{s} \rightarrow \mathcal{O}_{Q_{n}}^{t} \rightarrow E \rightarrow 0 .
$$

Furthermore, in [32], Miró-Roig gave a cohomological characterisation of vector bundles of the form $F_{n, r}=\operatorname{Ker}\left(\mathcal{O}_{\mathbb{P}^{n}}(-r)^{a_{0}} \rightarrow \mathcal{O}_{\mathbb{P}^{n}}\right)$, where $r \geq 1, n \geq 2$ and $a_{0}=\left(\begin{array}{c}r+n \\ n\end{array}\right)$. When $r \leq n$, the $F_{n, r}^{\vee}$ are Steiner bundles on $\mathbb{P}^{n}$ in the sense of Dolgachev and Kapranov.

In this paper we generalise the concept of Steiner bundle on $\mathbb{P}^{n}$ and on $Q_{n}$ to smooth irreducible algebraic varieties and we give a cohomological characterisation of this larger class of vector bundles. One of the main tools to prove our central result will be the Beilinson type spectral sequence. Beilinson's theorem stated in [4] became a fundamental tool for classifying vector bundles on projective spaces and, in particular, for their cohomological characterisation. The growing interest in this latter problem lead to the generalisation of Beilinson's result to other varieties such as hyperquadrics and Grassmannians [27, 26], to smooth projective varieties with a geometric collection [14] and more generally, to smooth projective varieties with an $m$-block collection [15]. The duality properties of an $m$-block collection and its dual will also play an important role for proving the referred cohomological characterisation.

The paper is organised as follows. Section 1 provides preliminary material such as the notions of $m$-block collections and their mutations, dual $m$-block collections, as well as the Beilinson type spectral sequence for smooth projective varieties with an $m$-block collection.

In section 2 we first define Steiner bundles of type $\left(F_{0}, F_{1}\right)$ on a smooth irreducible algebraic variety. We give some examples and, in particular, we see that Steiner bundles of type $\left(F_{0}, F_{1}\right)$ include the Steiner bundles defined so far. We then prove our main result. Namely, we fix an $n$-dimensional smooth irreducible projective variety $X$ with an $n$-block collection $\mathscr{B}$ of locally free sheaves generating $D^{b}\left(\mathcal{O}_{X}-\bmod \right)$, and we give a cohomological 
characterisation of Steiner bundles of type $\left(F_{0}, F_{1}\right)$ on $X$, provided that $F_{0}$ and $F_{1}$ belong to two different blocks of $\Re$ (Theorem 2.4). The theorem allows us to deduce that extensions of Steiner bundles of type $\left(F_{0}, F_{1}\right)$ are again Steiner bundles of the same type. We then apply our main theorem to characterise cohomologically some Steiner bundles of type $\left(F_{0}, F_{1}\right)$ on $\mathbb{P}^{n}$ and $Q_{n}$. More precisely, first we characterise cohomologically Steiner bundles of type $\left(O_{\mathbb{P}^{n}}(a), \bigcirc_{\mathbb{P}^{n}}(b)\right)$ and $\left(\Omega_{\mathbb{P}^{n}}^{p}(p), \mathcal{O}_{\mathbb{P}^{n}}\right)$ on $\mathbb{P}^{n}$, with $0 \leq a<b \leq n$ and $1 \leq p \leq n$. Then, when $n \geq 3$ is odd, we characterise cohomologically Steiner bundles of type $\left({ }^{0} Q_{n}(a), \mathcal{O}_{Q_{n}}(b)\right)$ and $\left({ }_{Q_{n}}(c), \Sigma(n-1)\right)$ on $Q_{n}$, with $0 \leq a<b \leq n$ and $0 \leq c \leq n-1$. When $n \geq 2$ is even, we characterise cohomologically Steiner bundles of type $\left({ }^{\circ} Q_{n}(a), \mathscr{O}_{Q_{n}}(b)\right)$ and $\left(\Sigma \pm(-n), \mathbb{O}_{Q_{n}}(c)\right)$ on $Q_{n}$, with $-n+1 \leq a<b \leq 0$ and $-n+1 \leq c \leq 0$.

\section{Preliminaries}

Let $X$ be a smooth projective variety over an algebraically closed field $K$ of characteristic 0 and let $\mathscr{D}=D^{b}\left(\mathscr{O}_{X}-\bmod \right)$ be the bounded derived category of the abelian category of coherent sheaves of $\mathrm{O}_{X}$-modules on $X$.

Notation 1.1. For any pair of objects $A, B \in \mathscr{D}$, we will denote

$$
\operatorname{Hom}_{\mathscr{D}}^{\bullet}(A, B)=\bigoplus_{k \in \mathbb{Z}} \operatorname{Ext}_{\mathscr{D}}^{k}(A, B)
$$

and $\operatorname{Hom}_{\mathscr{D}}^{\times \bullet}(A, B)$ the dual of $\operatorname{Hom}_{\mathscr{D}}^{\bullet}(A, B)$.

The shift functor will be denoted by $E \rightarrow E[p]$. When $E$ is a complex this functor is defined by $(E[p])^{j}=E^{p+j}$.

We will identify a coherent sheaf $F$ on $X$ with the object $0 \rightarrow F \rightarrow 0 \in \mathscr{D}$, the complex concentrated in degree 0 .

Definition 1.2. An object $E \in \mathscr{D}$ is called exceptional if

$$
\operatorname{Ext}_{\mathscr{D}}^{p}(E, E)= \begin{cases}K & \text { if } p=0 \\ 0 & \text { if } p \neq 0\end{cases}
$$

An ordered collection of objects of $\mathscr{D},\left(E_{1}, \ldots, E_{m}\right)$, is an exceptional collection if all objects $E_{i}$ are exceptional and

$$
\operatorname{Ext}_{\mathscr{P}}^{p}\left(E_{i}, E_{j}\right)=0 \text { for all } i>j \text { and all } p \in \mathbb{Z} .
$$

If, in addition,

$$
\operatorname{Ext}_{\mathscr{D}}^{p}\left(E_{j}, E_{i}\right)=0 \text { for all } j \leq i \text { and } p \neq 0,
$$

the collection $\left(E_{1}, \ldots, E_{m}\right)$ is called a strongly exceptional collection.

An ordered collection $\left(E_{1}, \ldots, E_{m}\right)$ of objects of $\mathscr{D}$ is a full (strongly) exceptional collection if it is a (strongly) exceptional collection such that $\mathscr{D}$ is generated by $E_{1}, \ldots, E_{m}$ 
i.e., any full triangulated subcategory containing all objects $E_{i}$ is equivalent to $\mathscr{D}$ (via the inclusion).

\section{Example 1.3.}

(1) The collections $\left(\mathscr{O}_{\mathbb{P}^{n}}, \widehat{O}_{\mathbb{P}^{n}}(1), \ldots, \mathscr{O}_{\mathbb{P}^{n}}(n)\right)$ and $\left(\Omega_{\mathbb{P}^{n}}^{n}(n), \ldots, \Omega_{\mathbb{P}^{n}}^{1}(1), \mathscr{O}_{\mathbb{P}^{n}}\right)$ of locally free sheaves on $\mathbb{P}^{n}$ are both strongly exceptional collections. In [4] Beilinson proved that they are full.

(2) Let $Q_{n} \subset \mathbb{P}^{n+1}$ be a smooth hyperquadric. If $n$ is odd then the collection $\left(\Sigma(-n), \mathcal{O}_{Q_{n}}(-n+1), \ldots, \mathcal{O}_{Q_{n}}\right)$, where $\Sigma$ is the Spinor bundle on $Q_{n}$, is a full strongly exceptional collection of locally free sheaves on $Q_{n}$. If $n$ is even, the collection $\left(\Sigma_{+}(-n), \Sigma_{-}(-n), \mathrm{O}_{Q_{n}}(-n+1), \ldots, \mathcal{O}_{Q_{n}}\right)$, where $\Sigma_{+}$and $\Sigma_{-}$are the Spinor bundles on $Q_{n}$, is a full strongly exceptional collection of locally free sheaves on $Q_{n}$ (see [27], Proposition 4.9 and its proof, and [34], Theorem 2.1).

(3) Consider the Grassmannian $X=\operatorname{Gr}(k, n)$ of $k$-dimensional subspaces of an $n$-dimensional vector space $V$. There exists a canonical exact sequence

$$
0 \rightarrow \mathscr{Y} \rightarrow \mathrm{O}_{X}^{n} \rightarrow 2 \rightarrow 0
$$

where $\mathscr{Y}$ is the tautological $k$-bundle and 2 the quotient bundle.

Let $\Sigma^{\alpha} \mathscr{Y}$ denote the space of the irreducible representations of the group GL( $\left.\mathscr{Y}\right)$ with highest weight $\alpha=\left(\alpha_{1}, \ldots, \alpha_{s}\right)$ and let $|\alpha|=\sum_{i=1}^{s} \alpha_{i}$. Denote by $A(k, n)$ the set of locally free sheaves $\Sigma^{\alpha} \mathscr{Y}$ on $\operatorname{Gr}(k, n)$, where $\alpha$ runs over Young diagrams fitting inside a $k \times(n-k)$ rectangle. Set $\rho(k, n):=\sharp A(k, n)$. By [26], Proposition 2.2 and 1.4, $A(k, n)$ can be ordered in such a way that we obtain a full strongly exceptional collection $\left(E_{1}, \ldots, E_{\rho(k, n)}\right)$ of locally free sheaves on $\operatorname{Gr}(k, n)$.

Before introducing the fundamental concept of $n$-block collection we need to define mutations of exceptional collections of objects of $\mathscr{D}$. The use of mutations is essentially a way of producing new exceptional collections.

Definition 1.4. Let $X$ be a smooth projective variety and $\sigma=(A, B)$ be an exceptional pair of objects of $\mathscr{D}$. Consider the object $L_{A} B$ that completes the canonical morphism $\operatorname{Hom}_{\mathscr{D}}^{\bullet}(A, B) \otimes A \rightarrow B$ to a distinguished triangle

$$
L_{A} B \rightarrow \operatorname{Hom}_{\mathscr{D}}^{\bullet}(A, B) \otimes A \rightarrow B \rightarrow L_{A} B[1],
$$

and the object $R_{B} A$ that completes the canonical morphism $A \rightarrow \operatorname{Hom}_{\mathscr{D}}^{\times \bullet}(A, B) \otimes B$ to a distinguished triangle

$$
R_{B} A[-1] \rightarrow A \rightarrow \operatorname{Hom}_{\mathscr{D}}^{\times \bullet}(A, B) \otimes B \rightarrow R_{B} A .
$$

A left mutation of the pair $\sigma=(A, B)$ is the pair

$$
L_{A} \sigma=\left(L_{A} B, A\right)=(L B, A)
$$


and a right mutation of the pair $\sigma=(A, B)$ is the pair

$$
R_{A} \sigma=\left(B, R_{B} A\right)=(B, R A) .
$$

Example 1.5. Consider the exceptional pair $\left(O_{\mathbb{P}^{n}}, O_{\mathbb{P}^{n}}(1)\right)$ of objects in the bounded derived

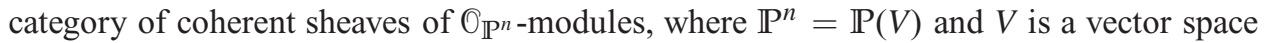
over $K$ of dimension $n+1$. Since

$$
\operatorname{Hom}_{\mathscr{D}}^{\bullet}\left(\bigcirc_{\mathbb{P}^{n}}, \bigcirc_{\mathbb{P}^{n}}(1)\right)=\operatorname{Ext}_{\mathscr{D}}^{0}\left(\bigcirc_{\mathbb{P}^{n}}, \bigcirc_{\mathbb{P}^{n}}(1)\right) \simeq V,
$$

then $R_{\mathscr{O}^{n}(1)} \bigcirc_{\mathbb{P}^{n}}$ is the element defined by the sequence

$$
0 \rightarrow \mathrm{O}_{\mathbb{P}^{n}} \rightarrow \operatorname{Hom}_{\mathscr{D}}^{\times \bullet}\left(\bigcirc_{\mathbb{P}^{n}}, \widehat{O}_{\mathbb{P}^{n}}(1)\right) \otimes \mathrm{O}_{\mathbb{P}^{n}}(1) \rightarrow R_{\bigcirc_{\mathbb{P}}(1)} \bigcirc_{\mathbb{P}^{n}} \rightarrow 0
$$

which in this case is nothing but the Euler sequence up to a twist,

$$
0 \rightarrow 0_{\mathbb{P}^{n}} \rightarrow \mathcal{O}_{\mathbb{P}^{n}}(1)^{n+1} \rightarrow R_{\mathscr{O}_{\mathbb{P}}^{n}(1)} \bigcirc_{\mathbb{P}^{n}} \rightarrow 0 .
$$

Hence, $R_{\mathscr{O}_{\mathbb{P}}^{n}(1)} \bigcirc_{\mathbb{P}^{n}}=T_{\mathbb{P}^{n}}$ and the right mutation of the pair $\left(\bigcirc_{\mathbb{P}^{n}}, \bigcirc_{\mathbb{P}^{n}}(1)\right)$ is the pair $\left(\bigcirc_{\mathbb{P}^{n}}(1), T_{\mathbb{P}^{n}}\right)$.

Definition 1.6. Let $X$ be a smooth projective variety. If $\sigma=\left(E_{0}, \ldots, E_{m}\right)$ is an exceptional collection of objects of $\mathscr{D}$ then a left mutation $L_{i} \sigma$ of $\sigma, 1 \leq i \leq m$, is the collection obtained by replacing the $i$-th pair of subsequent elements $\left(E_{i-1}, E_{i}\right)$ by its left mutation $\left(L_{E_{i-1}} E_{i}, E_{i-1}\right)$ :

$$
L_{i} \sigma=L_{E_{i-1}} \sigma=\left(E_{0}, \ldots, L_{E_{i-1}} E_{i}, E_{i-1}, \ldots, E_{m}\right)
$$

Similarly, a right mutation $R_{i} \sigma$ of $\sigma, 1 \leq i \leq m$, is the collection obtained by replacing the $i$-th pair of subsequent elements $\left(E_{i-1}, E_{i}\right)$ by its right mutation $\left(E_{i}, R_{E_{i}} E_{i-1}\right)$ :

$$
R_{i} \sigma=R_{E_{i-1}} \sigma=\left(E_{0}, \ldots, E_{i}, R_{E_{i}} E_{i-1}, \ldots, E_{m}\right)
$$

If $X$ is a smooth projective variety and $\sigma=\left(E_{0}, \ldots, E_{m}\right)$ is an exceptional collection of objects of $\mathscr{D}$ then any mutation of $\sigma$ is again an exceptional collection of objects of $\mathscr{D}$ (see [21], Proposition 2.4.2). Furthermore, when $\sigma$ is a full collection (i.e. when $E_{0}, \ldots, E_{m}$ generate the derived category $\mathscr{D}$ ) then any mutation of $\sigma$ is also full (see [20]). Nevertheless, a mutation of a strongly exceptional collection is not necessarily a strongly exceptional collection. For instance, let $X=\mathbb{P}^{1} \times \mathbb{P}^{1}$ be the smooth quadric surface in $\mathbb{P}^{3}$ and consider the full strongly exceptional collection $\sigma=\left(\bigcirc_{X}, \mathscr{O}_{X}(1,0), \bigcirc_{X}(0,1), \bigcirc_{X}(1,1)\right)$ of line bundles on $X$. We have

$$
\operatorname{Hom}_{\mathscr{D}}^{\bullet}\left(\mathrm{O}_{X}(0,1), \mathscr{O}_{X}(1,1)\right) \simeq \operatorname{Hom}_{\mathscr{D}}\left(\mathrm{O}_{X}(0,1), \mathscr{O}_{X}(1,1)\right)
$$


and thus the exact sequence

$$
0 \rightarrow L_{\mathscr{O}_{X}(0,1)} \mathscr{O}_{X}(1,1) \rightarrow \operatorname{Hom}_{\mathscr{D}}\left(\mathscr{O}_{X}(0,1), \mathscr{O}_{X}(1,1)\right) \otimes \mathscr{O}_{X}(0,1) \rightarrow \mathscr{O}_{X}(1,1) \rightarrow 0
$$

determines that $L_{\mathscr{O}_{X}(0,1)} \mathcal{O}_{X}(1,1) \simeq \mathbb{O}_{X}(-1,1)$ ([21], Example 4.5.2). Therefore, a left mutation of $\sigma$ is the collection $L_{3} \sigma=\left(\mathcal{O}_{X}, O_{X}(1,0), O_{X}(-1,1), O_{X}(0,1)\right)$ and this is no longer a strongly exceptional collection of line bundles on $X$ since $\operatorname{Ext}^{1}\left(O_{X}(1,0), O_{X}(-1,1)\right) \simeq$ $H^{1}\left(\mathbb{O}_{\mathbb{P}^{1}}(-3)\right) \simeq K^{2} \neq 0$.

Notation 1.7. Let $X$ be a smooth projective variety and let $\sigma=\left(E_{0}, \ldots, E_{m}\right)$ be an exceptional collection of objects of $\mathscr{D}$. We will use the following notation: for any $0 \leq i \leq m$, $1 \leq j \leq m$ and $i+j \leq m$,

$$
\begin{aligned}
& R^{(0)} E_{i}=E_{i}, \\
& R^{(j)} E_{i}=R^{(j-1)} R E_{i}=R_{E_{i+j}} \cdots R_{E_{i+2}} R_{E_{i+1}} E_{i}=R_{E_{i+j} \cdots E_{i+2} E_{i+1}} E_{i} .
\end{aligned}
$$

For any $1 \leq i, j \leq m$ and $i+j \leq m$,

$$
R_{E_{i-1}}^{(0)} \sigma=\sigma, \quad R_{E_{i-1}}^{(j)} \sigma=R_{R E_{i-1}}^{(j-1)} R_{E_{i-1}} \sigma .
$$

For composition of left mutations the notation is similar, that is, for any $0 \leq i \leq m$, $1 \leq j \leq m$ and $i-j \geq 0$,

$$
\begin{aligned}
& L^{(0)} E_{i}=E_{i}, \\
& L^{(j)} E_{i}=L^{(j-1)} L E_{i}=L_{E_{i-j}} \cdots L_{E_{i-2}} L_{E_{i-1}} E_{i}=L_{E_{i-j} \cdots E_{i-2} E_{i-1}} E_{i} .
\end{aligned}
$$

For any $1 \leq i, j \leq m$ and $i-j \geq 0$,

$$
L_{E_{i-1}}^{(0)} \sigma=\sigma, \quad L_{E_{i-1}}^{(j)} \sigma=L_{L E_{i-1}}^{(j-1)} L_{E_{i-1}} \sigma .
$$

Next we introduce the important concepts of blocks and $m$-block collections. They were first defined by Karpov and Nogin in [28] (see also [22]) and since then they have played an essential role, for example, for Costa and Miró-Roig's classification of vector bundles on multiprojective spaces $[15,13]$ or for the study of exceptional collections of coherent sheaves over Del Pezzo surfaces [28].

Definition 1.8. An exceptional collection $\left(E_{0}, \ldots, E_{m}\right)$ of objects of $\mathscr{D}$ is a block if $\operatorname{Ext}_{\mathscr{D}}^{p}\left(E_{j}, E_{i}\right)=0$ for all $p$ and all $i \neq j$.

An $m$-block collection of type $\left(\alpha_{0}, \ldots, \alpha_{m}\right)$ of objects of $\mathscr{D}$ is an exceptional collection

$$
\mathscr{B}=\left(\mathscr{E}_{0}, \ldots, \mathscr{E}_{m}\right)=\left(E_{1}^{0}, \ldots, E_{\alpha_{0}}^{0}, E_{1}^{1}, \ldots, E_{\alpha_{1}}^{1}, \ldots, E_{1}^{m}, \ldots, E_{\alpha_{m}}^{m}\right),
$$

where each subcollection $\mathscr{E}_{j}=\left(E_{1}^{j}, \ldots, E_{\alpha_{j}}^{j}\right)$ is a block.

Note that an exceptional collection $\left(E_{0}, \ldots, E_{m}\right)$ is an $m$-block collection of type $(1, \ldots, 1)$. 


\section{Example 1.9.}

(1) As seen in Example $1.3(1)$, the collections $\left(0_{\mathbb{P}^{n}}, \bigcirc_{\mathbb{P}^{n}}(1), \ldots, \widehat{O}_{\mathbb{P}^{n}}(n)\right)$ and $\left(\Omega_{\mathbb{P}^{n}}^{n}(n), \ldots\right.$, $\left.\Omega_{\mathbb{P}^{n}}^{1}(1), O_{\mathbb{P}^{n}}\right)$ are exceptional collections of locally free sheaves on $\mathbb{P}^{n}$. Therefore, they are $n$-block collections of type $(1, \ldots, 1)$.

(2) Let $Q_{n} \subset \mathbb{P}^{n+1}$ be a smooth hyperquadric. Recalling Example 1.3 (2), we see that if $n$ is odd, the collection $\left(\mathscr{E}_{0}, \ldots, \mathscr{E}_{n}\right)$ defined by

$$
\mathscr{E}_{0}=\Sigma(-n), \quad \mathscr{E}_{j}=\mathscr{O}_{Q_{n}}(-n+j), \text { for } 1 \leq j \leq n,
$$

is an $n$-block collection of locally free sheaves on $Q_{n}$ of type $(1, \ldots, 1)$. If $n$ is even, the collection $\left(\mathscr{E}_{0}, \ldots, \mathscr{E}_{n}\right)$ defined by

$$
\mathscr{E}_{0}=\left(\Sigma_{+}(-n), \Sigma_{-}(-n)\right), \quad \mathscr{E}_{j}=\mathscr{O}_{Q_{n}}(-n+j), \text { for } 1 \leq j \leq n
$$

is an $n$-block collection of locally free sheaves on $Q_{n}$ of type $(2,1, \ldots, 1)$.

(3) Let $\mathscr{B}=\left(\mathscr{E}_{0}, \ldots, \mathscr{E}_{n}\right), \mathscr{E}_{i}=\left(E_{1}^{i}, \ldots, E_{\alpha_{i}}^{i}\right)$, be an $n$-block collection of coherent sheaves generating $\mathscr{D}$. Then, the collection $\left(\mathscr{E}_{n}, \ldots, \mathscr{E}_{0}^{\vee}\right)$, where $\mathscr{E}_{i} \vee=\left(\left(E_{\alpha_{i}}^{i}\right)^{\vee}, \ldots,\left(E_{1}^{i}\right)^{\vee}\right)$, and the collection $\left(\mathscr{E}_{0} \otimes L, \ldots, \mathscr{E}_{n} \otimes L\right)$, where $\mathscr{E}_{i} \otimes L=\left(E_{1}^{i} \otimes L, \ldots, E_{\alpha_{i}}^{i} \otimes L\right)$ and $L$ is a line bundle, are still $n$-block collections of coherent sheaves generating $\mathscr{D}$.

(4) Consider the Grassmannian $X=\operatorname{Gr}(k, n)$ of $k$-dimensional subspaces of an $n$-dimensional vector space $V$. We saw in Example $1.3(3)$ that $A(k, n)$ can be ordered in such a way that $\sigma=\left(E_{1}, \ldots, E_{\rho(k, n)}\right)$ is a full strongly exceptional collection of locally free sheaves on $\operatorname{Gr}(k, n)$. In [27] Kapranov showed that $\operatorname{Hom}\left(\Sigma^{\alpha} \mathscr{Y}, \Sigma^{\beta} \mathscr{Y}\right) \neq 0$ only if $\alpha_{i} \geq \beta_{i}$ for all $i$. So, packing in the same block $\mathscr{E}_{r}$ the bundles $\Sigma^{\alpha} \mathscr{S} \in \sigma$ with $|\alpha|=k(n-k)-r$ and taking into account that $0 \leq|\alpha| \leq k(n-k)$ we obtain a $k(n-k)$-block collection of vector bundles on $X$,

$$
\sigma=\left(E_{1}, \ldots, E_{\rho(k, n)}\right)=\left(\mathscr{E}_{0}, \ldots, \mathscr{E}_{k(n-k)}\right) .
$$

Other examples of $m$-block collection can be found in [15] or [28].

Remark 1.10. An $m$-block collection $\mathscr{B}=\left(\mathscr{E}_{0}, \ldots, \mathscr{E}_{m}\right)$ is, by definition, an exceptional collection. Hille proved ([22], Theorem 1) that $\mathscr{B}$ is, furthermore, a strongly exceptional collection.

We now define mutations of block collections.

Definition 1.11. Let $X$ be a smooth projective variety and consider a 1-block collection $(\mathscr{E}, \mathscr{F})=\left(E_{1}, \ldots, E_{n}, F_{1}, \ldots, F_{m}\right)$ of type $(n, m)$ of objects of $\mathscr{D}$. A left mutation of $F_{j}$ by $\mathscr{E}$ is the object

$$
L_{\mathscr{E}} F_{j}=L_{E_{1}} L_{E_{2}} \cdots L_{E_{n}} F_{j}=L_{E_{1} E_{2} \cdots E_{n}} F_{j} .
$$


A right mutation of $E_{j}$ by $\mathscr{F}$ is the object

$$
R_{\mathscr{F}} E_{j}=R_{F_{m}} R_{F_{m-1}} \cdots R_{F_{1}} E_{j}=R_{F_{m} F_{m-1} \cdots F_{1}} E_{j} .
$$

A left mutation of the pair $(\mathscr{E}, \mathscr{F})$ is the pair $\left(L_{\mathscr{E}} \mathscr{F}, \mathscr{E}\right)$, where

$$
L_{\mathscr{E}} \mathscr{F}=\left(L_{\mathscr{E}} F_{1}, \ldots, L_{\mathscr{E}} F_{m}\right)
$$

and a right mutation of the pair $(\mathscr{E}, \mathscr{F})$ is the pair $\left(\mathscr{F}, R_{\mathscr{F}} \mathscr{E}\right)$, where

$$
R_{\mathscr{F}} \mathscr{E}=\left(R_{\mathscr{F}} E_{1}, \ldots, R_{\mathscr{F}} E_{n}\right)
$$

Let us remark that given a 1 -block collection $(\mathscr{E}, \mathscr{F})$ the collections $L_{\mathscr{E}} \mathscr{F}$ and $R_{\mathscr{F}} \mathscr{E}$ are blocks and the pairs $\left(L_{\mathscr{E}} \mathscr{F}, \mathscr{E}\right)$ and $\left(\mathscr{F}, R_{\mathscr{F}} \mathscr{E}\right)$ are 1-block collections (see [28], 2.1).

Notation 1.12. If $\mathscr{B}=\left(\mathscr{E}_{0}, \ldots, \mathscr{E}_{m}\right)$ is an $m$-block collection we will denote the composition of mutations of blocks as follows:

$$
\begin{aligned}
& R^{(0) \mathscr{E}_{i}}=\mathscr{E}_{i}, \\
& R^{(j) \mathscr{E}_{i}}=R^{(j-1)} R_{\mathscr{E}_{i}}=R_{\mathscr{E}_{i+j}} \cdots R_{\mathscr{E}_{i+2}} R_{\mathscr{E}_{i+1}} \mathscr{E}_{i}=R_{\mathscr{E}_{i+j}} \cdots \mathscr{E}_{i+2} \mathscr{E}_{i+1} \mathscr{E}_{i},
\end{aligned}
$$

for all $1 \leq j \leq m, 0 \leq i \leq m$ and $i+j \leq m$, and

$$
\begin{aligned}
& L^{(0) \mathscr{E}_{i}}=\mathscr{E}_{i}, \\
& L^{(j) \mathscr{E}_{i}}=L^{(j-1)} L \mathscr{E}_{i}=L_{\mathscr{E}_{i-j}} \cdots L_{\mathscr{E}_{i-2}} L_{\mathscr{E}_{i-1}} \mathscr{E}_{i}=L_{\mathscr{E}_{i-j}} \cdots \mathscr{E}_{i-2} \mathscr{E}_{i-1} \mathscr{E}_{i},
\end{aligned}
$$

for all $1 \leq j \leq m, 0 \leq i \leq m$ and $i-j \geq 0$.

The following definition will be central in what follows.

Definition 1.13. Let $\mathscr{B}=\left(\mathscr{E}_{0}, \ldots, \mathscr{E}_{m}\right)$ be an $m$-block collection of type $\left(\alpha_{0}, \ldots, \alpha_{m}\right)$. The left dual m-block collection of $\mathscr{B}$ is the $m$-block collection

$$
\mathscr{H}=\left(R^{(0)} \mathscr{E}_{m}, R^{(1)} \mathscr{E}_{m-1}, \ldots, R^{(m)} \mathscr{E}_{0}\right),
$$

where by definition

$$
\begin{aligned}
R^{(i) \mathscr{E}_{m-i}} & =\left(R^{(i)} E_{1}^{m-i}, \ldots, R^{(i)} E_{\alpha_{m-i}}^{m-i}\right) \\
& =\left(R_{\mathscr{E}_{m}} \mathscr{E}_{m-1} \cdots \mathscr{E}_{m-i+1} E_{1}^{m-i}, \ldots, R_{\mathscr{E}_{m}} \mathscr{E}_{m-1} \ldots \mathscr{E}_{m-i+1} E_{\alpha_{m-i}}^{m-i}\right) .
\end{aligned}
$$

Similarly, one defines the right dual m-block collection of $\mathscr{B}$ as the $m$-block collection

$$
\mathscr{H}=\left(L^{(m)} \mathscr{E}_{m}, L^{(m-1)} \mathscr{E}_{m-1}, \ldots, L^{(1)} \mathscr{E}_{1}, L^{(0)} \mathscr{E}_{0}\right),
$$

where

$$
\begin{aligned}
L^{(i) \mathscr{E}_{i}} & =\left(L^{(i)} E_{1}^{i}, \ldots, L^{(i)} E_{\alpha_{i}}^{i}\right) \\
& =\left(L_{\mathscr{E}_{0}} \mathscr{E}_{1} \ldots \mathscr{E}_{i-1} E_{1}^{i}, \ldots, L_{\mathscr{E}_{0} \mathscr{E}_{1} \ldots \mathscr{E}_{i-1}} E_{\alpha_{i}}^{i}\right) .
\end{aligned}
$$


The next proposition will also be fundamental to prove our main theorem. For the sake of completeness we give a sketch of its proof (for a more detailed proof see [13], Proposition 3.9).

Proposition 1.14. If $X$ is a smooth projective variety with an m-block collection $\mathscr{B}=$ $\left(\mathscr{E}_{0}, \ldots, \mathscr{E}_{m}\right)$ of type $\left(\alpha_{0}, \ldots, \alpha_{m}\right)$ of objects of $\mathscr{D}$ which generate $\mathscr{D}$, left dual and right dual m-block collections of $\cong$ are unique up to isomorphism and they are determined by the following orthogonality conditions:

$$
\operatorname{Hom}_{\mathscr{D}}^{\bullet}\left(R^{(i)} E_{j}^{m-i}, E_{k}^{l}\right)=\operatorname{Hom}_{\mathscr{D}}^{\bullet}\left(E_{k}^{l}, L^{(i)} E_{j}^{i}\right)=0,
$$

with the only exception of

$$
\operatorname{Ext}_{\mathscr{D}}^{k}\left(R^{(k)} E_{i}^{m-k}, E_{i}^{m-k}\right)=\operatorname{Ext}_{\mathscr{D}}^{m-k}\left(E_{i}^{m-k}, L^{(m-k)} E_{i}^{m-k}\right)=K .
$$

Proof. We will make use of two facts about mutations of block collections (see also [13], Remark 3.7): given a 1 -block collection $(\mathscr{E}, \mathscr{F})=\left(E_{1}, \ldots, E_{n}, F_{1}, \ldots, F_{m}\right)$, one has:

$$
\operatorname{Hom}_{\mathscr{D}}^{\bullet}\left(E_{j}, L_{\mathscr{E}} F_{i}\right)=0 \text {, for all } 1 \leq j \leq n
$$

and

$$
\operatorname{Hom}_{\mathscr{\Phi}}^{\bullet}\left(E^{\prime}, H\right)=\operatorname{Hom}_{\mathscr{D}}^{\bullet}\left(E^{\prime}, L_{\mathscr{E}} H\right)[1],
$$

for any $H \in \mathscr{D}$ and any $E^{\prime}$ such that $\operatorname{Hom}_{\mathscr{D}}^{\bullet}\left(E^{\prime}, F_{j}\right)=0$, for all $1 \leq j \leq n$.

It follows from (1.3) that for all $0 \leq l \leq i-1$ and $1 \leq j \leq \alpha_{l}$,

$$
\operatorname{Hom}_{\mathscr{D}}^{\bullet}\left(E_{j}^{l}, L^{(i)} E_{k}^{i}\right)=\operatorname{Hom}_{\mathscr{D}}^{\bullet}\left(E_{j}^{l}, L_{\mathscr{E}_{0} \mathscr{E}_{1} \ldots \mathscr{E}_{i-1}} E_{k}^{i}\right)=0,
$$

On the other hand, since $\mathscr{B}$ is an exceptional collection we also have

$$
\operatorname{Hom}_{\mathscr{D}}^{\bullet}\left(E_{j}^{l}, E_{q}^{p}\right)=0, \quad 1 \leq j \leq \alpha_{l}, 1 \leq q \leq \alpha_{p},
$$

for all $i \leq l \leq m$ and $0 \leq p \leq i-1$. Hence, for any $i \leq l \leq m$ and $1 \leq j \leq \alpha_{l}$, recursively applying (1.4), we have

$$
\operatorname{Hom}_{\mathscr{\Phi}}^{\bullet}\left(E_{j}^{l}, L^{(i)} E_{k}^{i}\right)=\operatorname{Hom}_{\mathscr{D}}^{\bullet}\left(E_{j}^{l}, E_{k}^{i}\right)[-i]= \begin{cases}0 & l>i \\ K(\text { in degree } i) & l=i, j=k .\end{cases}
$$

Therefore, from (1.5) and (1.6) we deduce that the right dual $m$-block collection satisfies the orthogonality conditions (1.1) and (1.2). The argument for the left dual $m$-block collection is similar.

\section{Example 1.15.}

(1) Consider the $n$-block collection $\mathscr{B}=\left(\mathscr{O}_{\mathbb{P}^{n}}, \ldots, \mathcal{O}_{\mathbb{P}^{n}}(n)\right)$ on $\mathbb{P}^{n}=\mathbb{P}(V)$, where $V$ is a $K$-vector space of dimension $n+1$. Notice that $\mathscr{B}$ generates $\mathscr{D}$. We have the following exact sequence

$$
0 \rightarrow \wedge^{k-1} T_{\mathbb{P}^{n}} \stackrel{j_{k}}{\rightarrow} \wedge^{k} V \otimes \mathbb{O}_{\mathbb{P}^{n}}(k) \rightarrow \wedge^{k} T_{\mathbb{P}^{n}} \rightarrow 0,
$$


obtained by applying exterior powers to the Euler sequence

$$
0 \rightarrow \mathbb{O}_{\mathbb{P}^{n}} \rightarrow V \otimes \mathcal{O}_{\mathbb{P}^{n}}(1) \rightarrow T_{\mathbb{P}^{n}} \rightarrow 0
$$

and where the maps $j_{k}$ coincide with the canonical morphisms. Hence, for $j \geq 1$,

$$
R^{(j)} \widehat{O}_{\mathbb{P}^{n}}(n-j)=R_{\mathbb{O}_{\mathbb{P}^{n}(n)}} \cdots R_{\mathbb{O}_{\mathbb{P}^{n}}(n-j+1)} \mathcal{O}_{\mathbb{P}^{n}}(n-j)=\wedge^{j} T_{\mathbb{P}^{n}}(n-j),
$$

and thus, the left dual $n$-block collection of $\mathscr{B}$ is

$$
\mathscr{H}=\left(\mathbb{O}_{\mathbb{P}^{n}}(n), T_{\mathbb{P}^{n}}(n-1), \ldots, \wedge^{n} T_{\mathbb{P}^{n}}\right) .
$$

Note that the orthogonality relations (1.1) and (1.2) can be easily checked and are indeed satisfied.

(2) Consider the $n$-block collection $\mathscr{B}=\left(\Omega_{\mathbb{P}^{n}}^{n}(n), \ldots, \Omega_{\mathbb{P}^{n}}^{1}(1), \bigcirc_{\mathbb{P}^{n}}\right)$ on $\mathbb{P}^{n}$ of locally free sheaves generating $\mathscr{D}$. Its left dual $n$-block collection is the $n$-block collection

$\mathscr{H}=\left(O_{\mathbb{P}^{n}}, O_{\mathbb{P}^{n}}(1), \ldots, O_{\mathbb{P}^{n}}(n)\right)$. In fact, one can easily verify the orthogonality conditions (1.1) and (1.2).

(3) Consider the smooth hyperquadric $Q_{n} \subset \mathbb{P}^{n+1}, n \geq 2$. Recall (Example 1.9 (2)) that the collection $\mathscr{B}=\left(\mathscr{E}_{0}, \ldots, \mathscr{E}_{n}\right)$ defined by

$$
\begin{aligned}
& \mathscr{E}_{0}= \begin{cases}\left(\Sigma_{+}(-n), \Sigma_{-}(-n)\right) & \text { if } n \text { even } \\
\Sigma(-n) & \text { if } n \text { odd },\end{cases} \\
& \mathscr{E}_{j}=\mathscr{O}_{Q_{n}}(-n+j), 1 \leq j \leq n,
\end{aligned}
$$

is an $n$-block collection of locally free sheaves on $Q_{n}$ which generate $\mathscr{D}$. To determine its left dual $n$-block collection we set some notation first. We denote $\Omega^{j}=\Omega_{\mathbb{P}^{n+1}}^{j}$ and we define by induction the following locally free sheaves on $Q_{n}$ :

$$
\psi_{0}=\mathrm{O}_{Q_{n}}, \quad \psi_{1}=\Omega^{1}(1)_{\mid Q_{n}}
$$

and for $j \geq 2, \psi_{j}$ is defined as the unique non-splitting extension (note that $\left.\operatorname{Ext}^{1}\left(\psi_{j-2}, \Omega^{j}(j)_{\mid Q_{n}}\right) \simeq K\right)$,

$$
0 \rightarrow \Omega^{j}(j)_{Q_{n}} \rightarrow \psi_{j} \rightarrow \psi_{j-2} \rightarrow 0 .
$$

Notice that $\psi_{j}=\psi_{j+2}$ for $j \geq n$. These sheaves $\psi_{j}$ on $Q_{n}$ were first defined by Kapranov in order to describe the bounded derived category $\mathscr{D}\left(\mathbb{O}_{Q_{n}}-\right.$ mod $)$.

The left dual $n$-block collection $\mathscr{H}$ of $\mathscr{B}$ is given as follows: if $n$ is odd then $\mathscr{H}=$ $\left(\psi_{0}^{\vee}, \ldots, \psi_{n-1}^{\vee}, \Sigma\right)$, and if $n$ is even $\mathscr{H}=\left(\psi_{0}^{\vee}, \ldots, \psi_{n-1}^{\vee}, \Sigma_{+}, \Sigma_{-}\right)$(see [27]).

(4) Consider the smooth hyperquadric $Q_{n} \subset \mathbb{P}^{n+1}$, with $n \geq 2$ odd, and the $n$-block collection $\mathscr{B}=\left({ }^{\circ} Q_{n}, \mathscr{O}_{Q_{n}}(1), \ldots, \mathscr{O}_{Q_{n}}(n-1), \Sigma(n-1)\right)$ of type $(1, \ldots, 1)$ of coherent sheaves on $Q_{n}$ which generate $\mathscr{D}$ (note that $\mathscr{B}$ is the dual of the $n$-block collection considered in the previous example and recall Example $1.9(3))$. The left dual $n$-block collection of $\mathscr{P}$ is the collection $\mathscr{H}=\left(\Sigma(n-1), \psi_{n-1}(n), \ldots, \psi_{0}(n)\right)$, as proved in [14], Proposition 4.2. 
In [15], Costa and Miró-Roig generalised Beilinson Theorem, stated in [4], to any smooth projective variety of dimension $n$ with an $n$-block collection of coherent sheaves on $X$ which generate $\mathscr{D}$. The Beilinson type spectral sequence theorem will be a fundamental tool for our work.

Theorem 1.16 (Beilinson type spectral sequence). Let $X$ be a smooth projective variety of dimension $n$ with an $n$-block collection $\mathscr{B}=\left(\mathscr{E}_{0}, \ldots, \mathscr{E}_{n}\right), \mathscr{E}_{i}=\left(E_{1}^{i}, \ldots, E_{\alpha_{i}}^{i}\right)$ of coherent sheaves on $X$ which generate $\mathscr{D}$.

For any coherent sheaf $F$ on $X$ there exist two spectral sequences situated in the square $-n \leq p \leq 0,0 \leq q \leq n$, with $E_{1}$-term

$$
{ }_{I} E_{1}^{p q}=\bigoplus_{i=1}^{\alpha_{p+n}} \operatorname{Ext}^{q}\left(R^{(-p)} E_{i}^{p+n}, F\right) \otimes E_{i}^{p+n}
$$

and

$$
{ }_{I I} E_{1}^{p q}=\bigoplus_{i=1}^{\alpha_{p+n}} \operatorname{Ext}^{q}\left(\left(E_{i}^{p+n}\right)^{\vee}, F\right) \otimes\left(R^{(-p)} E_{i}^{p+n}\right)^{\vee}
$$

and differentials $d_{r}^{p q}: E_{r}^{p, q} \rightarrow E_{r}^{p+r, q-r+1}$ which converge to

$$
{ }_{I} E_{\infty}^{j}={ }_{I I} E_{\infty}^{j}= \begin{cases}F & \text { if } j=0 \\ 0 & \text { if } j \neq 0\end{cases}
$$

Proof. See [15], Theorem 3.10.

\section{Steiner bundles and their cohomological characterisation}

Steiner bundles were first defined by Dolgachev and Kapranov in [16] as vector bundles $E$ on a projective space $\mathbb{P}^{n}$ that are the cokernel of an injective morphism $\mathcal{O}_{\mathbb{P}^{n}}(-1)^{s} \rightarrow \mathcal{O}_{\mathbb{P}^{n}}^{t}$. In [36] we defined Steiner bundles on the smooth hyperquadric $Q_{n} \subset \mathbb{P}^{n+1}$ as vector bundles defined by an exact sequence of type

$$
0 \rightarrow \mathrm{O}_{Q_{n}}(-1)^{s} \rightarrow \mathrm{O}_{Q_{n}}^{t} \rightarrow E \rightarrow 0
$$

We now generalise these definitions to vector bundles on a smooth irreducible algebraic variety.

Definition 2.1. A vector bundle $E$ on a smooth irreducible algebraic variety $X$ is called a Steiner bundle of type $\left(F_{0}, F_{1}\right)$ if it is defined by an exact sequence of the form

$$
0 \rightarrow F_{0}^{s} \stackrel{\varphi}{\rightarrow} F_{1}^{t} \rightarrow E \rightarrow 0
$$

where $s, t \geq 1$ and $\left(F_{0}, F_{1}\right)$ is an ordered pair of vector bundles on $X$ satisfying the following two conditions: 
(i) $\left(F_{0}, F_{1}\right)$ is strongly exceptional;

(ii) $F_{0}^{\vee} \otimes F_{1}$ is generated by global sections.

Remark 2.2. If $E$ is a Steiner bundle of type $\left(F_{0}, F_{1}\right)$ then $\operatorname{rk}(E) \geq n$. In fact, denoting by $r_{i}$ the rank of $F_{i}, i=0,1$, since $F_{0}^{\vee} \otimes F_{1}$ is generated by global sections then a general degeneracy locus,

$$
D_{s r_{0}-1}(\varphi)=\left\{x \in X \mid \operatorname{rank}(\varphi(x)) \leq s r_{0}-1\right\}
$$

is a subvariety of $X$ of codimension $t r_{1}-s r_{0}+1$ (see for example [10]). If $\mathrm{rk} E=t r_{1}-s r_{0}<$ $n$ then $t r_{1}-s r_{0}+1 \leq n$ and thus, $D_{s r_{0}-1}(\varphi) \neq \emptyset$. In particular, $\operatorname{coker} \varphi$ would not a be vector bundle, contradicting our assumption on $E$.

We now see some examples and also that the above definition generalises the Steiner bundles defined so far.

\section{Example 2.3.}

(1) Let $E$ be a vector bundle on $\mathbb{P}^{n}$ defined by an exact sequence

$$
0 \rightarrow 0_{\mathbb{P}^{n}}(a)^{s} \rightarrow \mathcal{O}_{\mathbb{P}^{n}}(b)^{t} \rightarrow E \rightarrow 0,
$$

where $1 \leq b-a \leq n$. Thus, $E$ is a Steiner bundle of type $\left(0_{\mathbb{P}^{n}}(a), O_{\mathbb{P}^{n}}(b)\right)$ on $\mathbb{P}^{n}$. When $a=-1$ and $b=0$ these vector bundles are nothing but the classical Steiner vector bundles as defined by Dolgachev and Kapranov.

Let $Q_{n} \subset \mathbb{P}^{n+1}, n \geq 3$ be the smooth hyperquadric. Then the restriction of the above sequence to $Q_{n}$ gives us the short exact sequence

$$
0 \rightarrow \mathrm{O}_{Q_{n}}(a)^{s} \rightarrow \mathrm{O}_{Q_{n}}(b)^{t} \rightarrow E_{\left.\right|_{Q_{n}}} \rightarrow 0,
$$

and we see that $E_{\left.\right|_{Q_{n}}}$ is a Steiner vector bundle of type $\left({ }^{0} Q_{n}(a),{ }^{0} Q_{n}(b)\right)$ on $Q_{n}$. When $a=-1$ and $b=0, E_{\left.\right|_{Q_{n}}}$ is a Steiner vector bundle according to [36].

(2) Consider a short exact sequence of vector bundles on $\mathbb{P}^{n}$ of the form

$$
0 \rightarrow \Omega_{\mathbb{P}^{n}}^{p}(p)^{s} \rightarrow \mathrm{O}_{\mathbb{P}^{n}}^{t} \rightarrow E \rightarrow 0,
$$

where $1 \leq p \leq n$. Then $E$ is a Steiner vector bundle of type $\left(\Omega_{\mathbb{P}^{n}}^{p}(p), \mathcal{O}_{\mathbb{P}^{n}}\right)$ on $\mathbb{P}^{n}$.

(3) Any short exact sequence of vector bundles on $\mathbb{P}^{n}$

$$
0 \rightarrow \mathbb{O}_{\mathbb{P}^{n}}(-1)^{s} \rightarrow \Omega_{\mathbb{P}^{n}}^{p}(p)^{t} \rightarrow E \rightarrow 0,
$$

where $0 \leq p \leq n-1$, defines a Steiner bundle $E$ of type $\left(0_{\mathbb{P}^{n}}(-1), \Omega_{\mathbb{P}^{n}}^{p}(p)\right)$ on $\mathbb{P}^{n}$. 
(4) Consider the smooth hyperquadric $Q_{n} \subset \mathbb{P}^{n+1}, n \geq 2$, and let $\Sigma_{( \pm)}$denote the Spinor bundle $\Sigma$ on $Q_{n}$ if $n$ is odd, and one of the Spinor bundles $\Sigma_{+}$or $\Sigma_{-}$on $Q_{n}$ if $n$ is even. Then if $E$ is a vector bundle defined by an exact sequence of the form

$$
0 \rightarrow \mathrm{O}_{Q_{n}}(a)^{s} \rightarrow \Sigma_{( \pm)}(n-1)^{t} \rightarrow E \rightarrow 0
$$

for some $0 \leq a \leq n-1, E$ is a Steiner bundle of type $\left(\bigcirc_{Q_{n}}(a), \Sigma_{( \pm)}(n-1)\right)$ on $Q_{n}$.

(5) The vector bundle $E$ on the smooth hyperquadric $Q_{n} \subset \mathbb{P}^{n+1}, n \geq 2$, defined by an exact sequence of the form

$$
0 \rightarrow \Sigma_{( \pm)}(-n)^{s} \rightarrow \mathrm{O}_{Q_{n}}(a)^{t} \rightarrow E \rightarrow 0
$$

where $-n+1 \leq a \leq 0$, is a Steiner bundle of type $\left(\Sigma_{( \pm)}(-n),{ }^{\circ} Q_{n}(a)\right)$ on $Q_{n}$.

In the present work we approach the problem of the cohomological characterisation of Steiner bundles of type $\left(F_{0}, F_{1}\right)$. Other properties of Steiner bundles of type $\left(F_{0}, F_{1}\right)$ were studied in [37]. In particular, we characterise generic exceptional and generic simple Steiner bundles of type $\left(F_{0}, F_{1}\right)$ and we prove that any exceptional Steiner bundle of type $\left(\mathrm{O}_{X}(-1), \mathrm{O}_{X}\right)$ on a smooth hypersurface $X \subset \mathbb{P}^{n}$ of degree $1 \leq d \leq n-1$ is stable.

We now prove our main result, that is, we give a cohomological characterisation of Steiner bundles $E$ of type $\left(F_{0}, F_{1}\right)$.

Theorem 2.4. Let $X$ be a smooth irreducible projective variety of dimension $n$ with an $n$-block collection $\mathscr{B}=\left(\mathscr{E}_{0}, \mathscr{E}_{1}, \ldots, \mathscr{E}_{n}\right), \mathscr{E}_{i}=\left(E_{1}^{i}, \ldots, E_{\alpha_{i}}^{i}\right)$, of locally free sheaves on $X$ which generate $\mathscr{D}$. Let $E_{i_{0}}^{a} \in \mathscr{E}_{a}, E_{j_{0}}^{b} \in \mathscr{E}_{b}$, where $0 \leq a<b \leq n$ and $1 \leq i_{0} \leq \alpha_{a}$, $1 \leq j_{0} \leq \alpha_{b}$, and let $E$ be a locally free sheaf on $X$.

Then $E$ is a Steiner bundle of type $\left(E_{i_{0}}^{a}, E_{j_{0}}^{b}\right)$ defined by an exact sequence of the form

$$
0 \rightarrow\left(E_{i_{0}}^{a}\right)^{s} \rightarrow\left(E_{j_{0}}^{b}\right)^{t} \rightarrow E \rightarrow 0
$$

if and only if $\left(E_{i_{0}}^{a}\right)^{\vee} \otimes E_{j_{0}}^{b}$ is generated by global sections and all $\mathrm{Ext}^{k}\left(R^{(m)} E_{i}^{n-m}, E\right)$ vanish, with the only exceptions of

$$
\begin{aligned}
& \operatorname{Ext}^{n-a-1}\left(R^{(n-a)} E_{i_{0}}^{a}, E\right)=K^{s}, \\
& \operatorname{Ext}^{n-b}\left(R^{(n-b)} E_{j_{0}}^{b}, E\right)=K^{t} .
\end{aligned}
$$

Proof. Suppose $E$ is a Steiner bundle of type $\left(E_{i_{0}}^{a}, E_{j_{0}}^{b}\right)$ defined by an exact sequence of the form

$$
0 \rightarrow\left(E_{i_{0}}^{a}\right)^{s} \rightarrow\left(E_{j_{0}}^{b}\right)^{t} \rightarrow E \rightarrow 0
$$

Then in particular $\left(E_{i_{0}}^{a}\right)^{\vee} \otimes E_{j_{0}}^{b}$ is generated by global sections. 
Applying the functor $\operatorname{Hom}\left(R^{(m)} E_{i}^{n-m},-\right)$ to the above sequence, where $0 \leq m \leq n$ and $1 \leq i \leq \alpha_{n-q}$, we obtain the exact sequence

$$
\begin{aligned}
& \cdots \rightarrow \operatorname{Hom}^{k}\left(R^{(m)} E_{i}^{n-m},\left(E_{i_{0}}^{a}\right)^{s}\right) \rightarrow \operatorname{Hom}^{k}\left(R^{(m)} E_{i}^{n-m},\left(E_{j_{0}}^{b}\right)^{t}\right) \rightarrow \\
& \left.\rightarrow \operatorname{Hom}^{k}\left(R^{(m)} E_{i}^{n-m}, E\right)\right) \rightarrow \operatorname{Hom}^{k+1}\left(R^{(m)} E_{i}^{n-m},\left(E_{i_{0}}^{a}\right)^{s}\right) \rightarrow \cdots
\end{aligned}
$$

The orthogonal conditions (1.1) and (1.2) of Proposition 1.14 now imply the following vanishing:

$$
\operatorname{Ext}^{k}\left(R^{(m)} E_{i}^{n-m},\left(E_{i_{0}}^{a}\right)^{s}\right)=\operatorname{Ext}^{k}\left(R^{(m)} E_{i}^{n-m},\left(E_{j_{0}}^{b}\right)^{t}\right)=0
$$

with the only exceptions of

$$
\operatorname{Ext}^{n-a}\left(R^{(n-a)} E_{i_{0}}^{a},\left(E_{i_{0}}^{a}\right)^{s}\right)=K^{s}, \quad \operatorname{Ext}^{n-b}\left(R^{(n-b)} E_{j_{0}}^{b},\left(E_{j_{0}}^{b}\right)^{t}\right)=K^{t}
$$

Since $a<b$ we thus deduce the vanishing of all $\operatorname{Ext}^{k}\left(R^{(m)} E_{i}^{n-m}, E\right)$, with the only exceptions of

$$
\operatorname{Ext}^{n-a-1}\left(R^{(n-a)} E_{i_{0}}^{a}, E\right)=K^{s} \text { and } \operatorname{Ext}^{n-b}\left(R^{(n-b)} E_{j_{0}}^{b}, E\right)=K^{t} .
$$

Conversely, suppose that all $\operatorname{Ext}^{k}\left(R^{(m)} E_{i}^{n-m}, E\right)$ vanish, the only exceptions being conditions (2.2) and (2.3). Recall that $\left(E_{i_{0}}^{a}, E_{j_{0}}^{b}\right)$ is a strongly exceptional pair, as observed in Remark 1.10. Since by hypothesis $\left(E_{i_{0}}^{a}\right)^{\vee} \otimes E_{j_{0}}^{b}$ is generated by global sections, we only need to see that there exists an exact sequence

$$
0 \rightarrow\left(E_{i_{0}}^{a}\right)^{s} \rightarrow\left(E_{j_{0}}^{b}\right)^{t} \rightarrow E \rightarrow 0
$$

To this end, we will apply Beilinson type spectral sequence. Indeed, by Theorem 1.16, there exists a Beilinson type spectral sequence situated in the square $0 \leq q \leq n,-n \leq p \leq 0$, with $E_{1}$-term

$$
E_{1}^{p q}=\bigoplus_{i=1}^{\alpha_{p+n}} \operatorname{Ext}^{q}\left(R^{(-p)} E_{i}^{p+n}, E\right) \otimes E_{i}^{p+n}
$$

and which converges to

$$
E_{\infty}^{i}= \begin{cases}E & \text { if } i=0 \\ 0 & \text { if } i \neq 0\end{cases}
$$

The vanishing of $\operatorname{Ext}^{k}\left(R^{(m)} E_{i}^{n-m}, E\right)$ and cohomological conditions (2.2) and (2.3) imply that the $E_{1}$-term looks like: 


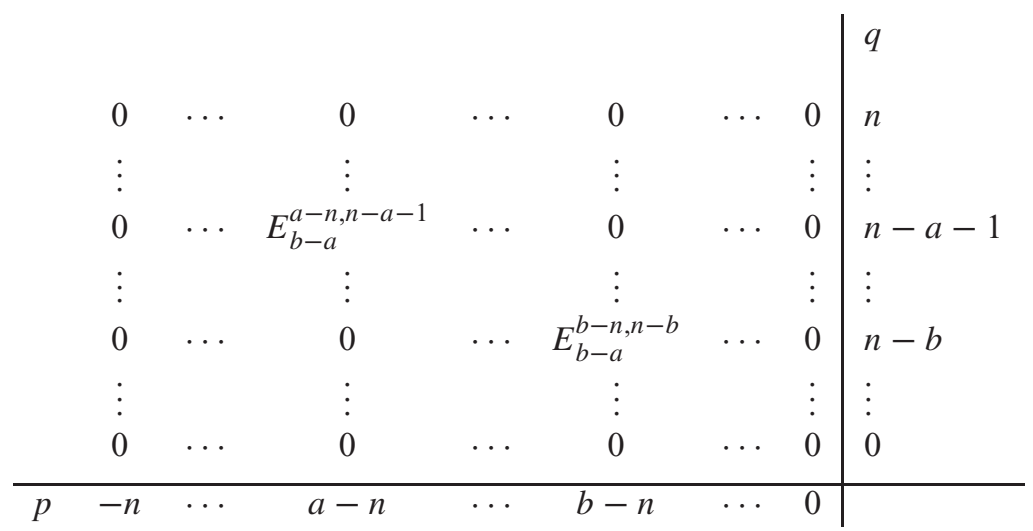

We thus deduce that the only non-vanishing differential is

$$
d_{b-a}^{a-n, n-a-1}: E_{b-a}^{a-n, n-a-1} \rightarrow E_{b-a}^{b-n, n-b}
$$

and that the spectral sequence collapses at the $E_{b-a+1}$-term. Therefore,

$$
\operatorname{ker}\left(d_{b-a}^{a-n, n-a-1}\right)=0, \quad E \simeq \operatorname{coker}\left(d_{b-a}^{a-n, n-a-1}\right),
$$

that is, there exists a short exact sequence

$$
0 \rightarrow E_{b-a}^{a-n, n-a-1} \rightarrow E_{b-a}^{b-n, n-b} \rightarrow E \rightarrow 0 .
$$

Now, cohomological condition (2.2) implies that

$$
\begin{aligned}
E_{b-a}^{a-n, n-a-1} & =\bigoplus_{i=1}^{\alpha_{a}} \operatorname{Ext}^{n-a-1}\left(R^{(n-a)} E_{i}^{a}, E\right) \otimes E_{i}^{a} \\
& =\operatorname{Ext}^{n-a-1}\left(R^{(n-a)} E_{i_{0}}^{a}, E\right) \otimes E_{i_{0}}^{a} \simeq\left(E_{i_{0}}^{a}\right)^{s},
\end{aligned}
$$

and cohomological condition (2.3) implies that

$$
\begin{aligned}
E_{b-a}^{b-n, n-b} & =\bigoplus_{j=1}^{\alpha_{b}} \operatorname{Ext}^{n-b}\left(R^{(n-b)} E_{j}^{b}, E\right) \otimes E_{j}^{b} \\
& =\operatorname{Ext}^{n-b}\left(R^{(n-b)} E_{j_{0}}^{b}, E\right) \otimes E_{j_{0}}^{b} \simeq\left(E_{j_{0}}^{b}\right)^{t} .
\end{aligned}
$$

Thus, $E$ is defined by an exact sequence

$$
0 \rightarrow\left(E_{i_{0}}^{a}\right)^{s} \rightarrow\left(E_{j_{0}}^{b}\right)^{t} \rightarrow E \rightarrow 0,
$$

and hence $E$ is a Steiner bundle of type $\left(E_{i_{0}}^{a}, E_{j_{0}}^{b}\right)$ on $X$.

The above theorem applies to any smooth projective variety $X$ of dimension $n$ which has an $n$-block collection which generates $\mathscr{D}$. This includes a large number of smooth projective varieties, namely projective spaces, hyperquadrics, Grassmannians, multiprojective spaces (see [13] for examples of $n$-block collections for all these cases), and also the Fano varieties $V_{5}, V_{22}$ or Fano threefolds of genus 7 (see [18], [19] and [8]). 
As a first corollary of the above theorem we see that extensions of Steiner bundles of type $\left(F_{0}, F_{1}\right)$ are Steiner bundles of the same type. More precisely, we have the following:

Corollary 2.5. Let $X$ be a smooth projective variety $X$ with an $n$-block collection $\mathscr{B}=$ $\left(\mathscr{E}_{0}, \mathscr{E}_{1}, \ldots, \mathscr{E}_{n}\right), \mathscr{E}_{i}=\left(E_{1}^{i}, \ldots, E_{\alpha_{i}}^{i}\right)$, of locally free sheaves on $X$ which generate $\mathscr{D}_{\text {. Let }}$ $E_{i_{0}}^{a} \in \mathscr{E}_{a}, E_{j_{0}}^{b} \in \mathscr{E}_{b}$, where $0 \leq a<b \leq n$ and $1 \leq i_{0} \leq \alpha_{a}, 1 \leq j_{0} \leq \alpha_{b}$.

If $E$ and $F$ are Steiner bundles of type $\left(E_{i_{0}}^{a}, E_{j_{0}}^{b}\right)$ on $X$ then any extension $G$ of $E$ by $F$,

$$
0 \rightarrow F \rightarrow G \rightarrow E \rightarrow 0
$$

is a Steiner bundle of type $\left(E_{i_{0}}^{a}, E_{j_{0}}^{b}\right)$ on $X$.

Proof. Since $E$ and $F$ are Steiner bundles of type $\left(E_{i_{0}}^{a}, E_{j_{0}}^{b}\right)$ then $\left(E_{i_{0}}^{a}\right)^{\vee} \otimes E_{j_{0}}^{b}$ is generated by global sections. Suppose $E$ and $F$ are, respectively, given by

$$
0 \rightarrow\left(E_{i_{0}}^{a}\right)^{s} \rightarrow\left(E_{j_{0}}^{b}\right)^{t} \rightarrow E \rightarrow 0 \quad \text { and } \quad 0 \rightarrow\left(E_{i_{0}}^{a}\right)^{s^{\prime}} \rightarrow\left(E_{j_{0}}^{b}\right)^{t^{\prime}} \rightarrow F \rightarrow 0 .
$$

From Theorem 2.4 we know that all $\operatorname{Ext}^{k}\left(R^{(m)} E_{i}^{n-m}, E\right)$ and $\operatorname{Ext}^{k}\left(R^{(m)} E_{i}^{n-m}, F\right)$ vanish, except in the following cases:

$$
\begin{array}{ll}
\operatorname{Ext}^{n-a-1}\left(R^{(n-a)} E_{i_{0}}^{a}, E\right)=K^{s}, & \operatorname{Ext}^{n-b}\left(R^{(n-b)} E_{j_{0}}^{b}, E\right)=K^{t}, \\
\operatorname{Ext}^{n-a-1}\left(R^{(n-a)} E_{i_{0}}^{a}, F\right)=K^{s^{\prime}}, & \operatorname{Ext}^{n-b}\left(R^{(n-b)} E_{j_{0}}^{b}, F\right)=K^{t^{\prime}} .
\end{array}
$$

Applying the functor $\operatorname{Hom}\left(R^{(m)} E_{i}^{n-m},-\right)$ to the sequence

$$
0 \rightarrow F \rightarrow G \rightarrow E \rightarrow 0
$$

we easily see that all $\operatorname{Ext}^{k}\left(R^{(m)} E_{i}^{n-m}, G\right)$ vanish, except

$$
\begin{aligned}
& \operatorname{Ext}^{n-a-1}\left(R^{(n-a)} E_{i_{0}}^{a}, G\right)=K^{s+s^{\prime}}, \\
& \operatorname{Ext}^{n-b}\left(R^{(n-b)} E_{j_{0}}^{b}, G\right)=K^{t+t^{\prime}} .
\end{aligned}
$$

Hence, by Theorem $2.4, G$ is a Steiner bundle of type $\left(E_{i_{0}}^{a}, E_{j_{0}}^{b}\right)$.

We now see a few applications of Theorem 2.4. Our first result concerns Steiner bundles of types $\left(\bigcirc_{\mathbb{P}^{n}}(a), \bigcirc_{\mathbb{P}^{n}}(b)\right)$ and $\left(\Omega_{\mathbb{P}^{n}}^{p}(p), \bigcirc_{\mathbb{P}^{n}}\right)$ on the projective space.

Corollary 2.6. Let $E$ be a vector bundle on $\mathbb{P}^{n}$. We have:

(a) E is a Steiner bundle of type $\left(\mathcal{O}_{\mathbb{P}^{n}}(a), \bigcirc_{\mathbb{P}^{n}}(b)\right)$, for some $0 \leq a<b \leq n$, given by an exact sequence

$$
0 \rightarrow \mathrm{O}_{\mathbb{P}^{n}}(a)^{s} \rightarrow \mathrm{O}_{\mathbb{P}^{n}}(b)^{t} \rightarrow E \rightarrow 0
$$

if and only if

(a1) $h^{n-a-1}\left(E \otimes \Omega_{\mathbb{P}^{n}}^{n-a}(-a)\right)=s$;

(a2) $h^{n-b}\left(E \otimes \Omega_{\mathbb{P}^{n}}^{n-b}(-b)\right)=t$; 
(a3) $\quad h^{k}\left(E \otimes \Omega_{\mathbb{P}^{n}}^{n-j}(-j)\right)=0$, for all $0 \leq k, j \leq n,(k, j) \neq(n-a-1, a)$ and $(k, j) \neq(n-b, b)$;

(b) E is a Steiner bundle of type $\left(\Omega_{\mathbb{P}^{n}}^{p}(p), \mathcal{O}_{\mathbb{P}^{n}}\right)$, for some $1 \leq p \leq n$, given by an exact sequence

$$
0 \rightarrow \Omega_{\mathbb{P}^{n}}^{p}(p)^{s} \rightarrow \mathrm{O}_{\mathbb{P}^{n}}^{t} \rightarrow E \rightarrow 0
$$

if and only if

(b1) $h^{p-1}(E(-p))=s$;

(b2) $h^{0}(E)=t$;

(b3) $h^{k}(E(-j))=0$, for all $0 \leq k, j \leq n,(k, j) \neq(p-1, p)$ and $(k, j) \neq(0,0)$.

Proof. Consider the $n$-block collection

$$
\mathscr{B}=\left(E_{1}^{0}, E_{1}^{1}, \ldots, E_{1}^{n}\right)=\left(\mathscr{O}_{\mathbb{P}^{n}}, \mathscr{O}_{\mathbb{P}^{n}}(1), \ldots, \mathscr{O}_{\mathbb{P}^{n}}(n)\right)
$$

of type $(1, \ldots, 1)$ of locally free sheaves on $\mathbb{P}^{n}$ generating $\mathscr{D}$ (recall Example $\left.1.9(1)\right)$. We saw in Example 1.15 (1) that the left dual $n$-block collection of $\mathscr{B}$ is

$$
\mathscr{H}=\left(\bigcirc_{\mathbb{P}^{n}}(n), T_{\mathbb{P}^{n}}(n-1), \ldots, \wedge^{j} T_{\mathbb{P}^{n}}(n-j), \ldots, \wedge^{n} T_{\mathbb{P}^{n}}\right) .
$$

Therefore, for all $0 \leq j \leq n$ we have

$$
\left(R^{(n-j)} E_{1}^{j}\right)^{\vee}=\left(R^{(n-j)} \mathcal{O}_{\mathbb{P}^{n}}(j)\right)^{\vee}=\left(\wedge^{n-j} T_{\mathbb{P}^{n}}(j)\right)^{\vee} \simeq \Omega_{\mathbb{P}^{n}}^{n-j}(-j) .
$$

Observe that $\left(E_{1}^{a}\right)^{\vee} \otimes E_{1}^{b}=\mathcal{O}_{\mathbb{P}^{n}}(a)^{\vee} \otimes \mathcal{O}_{\mathbb{P}^{n}}(b)=\mathcal{O}_{\mathbb{P}^{n}}(b-a)$ is generated by global sections since $b-a \geq 0$. So, by Theorem $2.4, E$ is a Steiner bundle of type $\left(E_{1}^{a}=O_{\mathbb{P}^{n}}(a), E_{1}^{b}=\right.$ $\left.\mathrm{O}_{\mathbb{P}^{n}}(b)\right)$, for some $0 \leq a<b \leq n$, given by

$$
0 \rightarrow \mathrm{O}_{\mathbb{P}^{n}}(a)^{s} \rightarrow \mathrm{O}_{\mathbb{P}^{n}}(a)^{t} \rightarrow E \rightarrow 0
$$

if and only if all $h^{k}\left(E \otimes\left(R^{(m)} E_{1}^{n-m}\right)^{\vee}\right)=h^{k}\left(E \otimes \Omega_{\mathbb{P}^{n}}^{n-j}(-j)\right)$ vanish, with the only exceptions of

$$
\begin{aligned}
& h^{n-a-1}\left(E \otimes\left(R^{(n-a)} E_{1}^{a}\right)^{\vee}\right)=h^{n-a-1}\left(E \otimes\left(\Omega_{\mathbb{P}^{n}}^{n-a}(-a)\right)=s,\right. \\
& h^{n-b}\left(E \otimes\left(R^{(n-b)} E_{1}^{b}\right)^{\vee}\right)=h^{n-b}\left(E \otimes \Omega_{\mathbb{P}^{n}}^{n-b}(-b)\right)=t .
\end{aligned}
$$

This proves (a).

The proof of (b) is analogous. In this case we consider the $n$-block collection $\mathscr{B}=$ $\left(\Omega_{\mathbb{P}^{n}}^{n}(n), \Omega_{\mathbb{P}^{n}}^{n-1}(n-1), \ldots, \Omega_{\mathbb{P}^{n}}^{1}(1), \mathcal{O}_{\mathbb{P}^{n}}\right)$ of type $(1, \ldots, 1)$ of locally free sheaves on $\mathbb{P}^{n}$ generating $\mathscr{D}$ (see Example 1.9 (1)). From Example 1.15 (2), we know that the left dual $n$-block collection of $\mathscr{B}$ is $\mathscr{H}=\left(O_{\mathbb{P}^{n}}, \mathscr{O}_{\mathbb{P}^{n}}(1), \ldots, \mathscr{O}_{\mathbb{P}^{n}}(n)\right)$. Note that the vector bundle $\left(E_{1}^{n-p}\right)^{\vee} \otimes E_{1}^{n}=\left(\Omega_{\mathbb{P}^{n}}^{p}(p)\right)^{\vee} \otimes \mathcal{O}_{\mathbb{P}^{n}}=\wedge^{p} T_{\mathbb{P}^{n}}(-p)$ is generated by global sections since we have a short exact sequence

$$
0 \rightarrow \bigwedge^{p-1} T_{\mathbb{P}^{n}}(-p) \rightarrow \bigwedge^{p} V \otimes \mathbb{O}_{\mathbb{P}^{n}} \rightarrow \bigwedge^{p} T_{\mathbb{P}^{n}}(-p) \rightarrow 0 .
$$

Then apply Theorem 2.4 to the pair $\left(E_{1}^{n-p}=\Omega_{\mathbb{P}^{n}}^{p}(p), E_{1}^{n}=O_{\mathbb{P}^{n}}\right)$. 
We now apply Theorem 2.4 to get a cohomological characterisation of some Steiner bundles of type $\left(F_{0}, F_{1}\right)$ on the smooth hyperquadric $Q_{n} \subset \mathbb{P}^{n+1}$. For general facts about Spinor bundles, and in particular their cohomology, we refer to [34], [27] and [14], whereas for basic facts about the $\psi_{i}$ bundles introduced in Example 1.15 (3), we refer to [27].

When $n$ is odd we have the following characterisation:

Corollary 2.7. Let $E$ be a vector bundle on the smooth hyperquadric $Q_{n} \subset \mathbb{P}^{n+1}$ with $n \geq 3$ odd. Then

(a) E is a Steiner bundle of type $\left({ }^{O} Q_{n}(a), \Sigma(n-1)\right)$, for some $0 \leq a \leq n-1$, given by an exact sequence

$$
0 \rightarrow \mathrm{O}_{Q_{n}}(a)^{S} \rightarrow \Sigma(n-1)^{t} \rightarrow E \rightarrow 0
$$

if and only if

(a1) $h^{n-a-1}\left(E \otimes \psi_{a}^{\vee}(-n)\right)=s$;

(a2) $h^{0}(E \otimes \Sigma(-n))=t$;

(a3) $h^{k}\left(E \otimes \psi_{j}^{\vee}(-n)\right)=h^{k}(E \otimes \Sigma(-n))=0$, for all $0 \leq k \leq n,(k, j) \neq(n-a-1, a)$.

(b) E is a Steiner bundle of type $\left({ }^{\circ} Q_{n}(a), O_{Q_{n}}(b)\right)$, for some $0 \leq a<b \leq n$, given by an exact sequence

$$
0 \rightarrow \mathrm{O}_{Q_{n}}(a)^{s} \rightarrow \mathrm{O}_{Q_{n}}(b)^{t} \rightarrow E \rightarrow 0
$$

if and only if

(b1) $h^{n-a-1}\left(E \otimes \psi_{a}^{\vee}(-n)\right)=s$;

(b2) $h^{n-b}\left(E \otimes \psi_{b}^{\vee}(-n)\right)=t$;

(b3) $h^{k}\left(E \otimes \psi_{j}^{\vee}(-n)\right)=h^{k}(E \otimes \Sigma(-n))=0$, for all $0 \leq k \leq n, 0 \leq j \leq n-1$, $(k, j) \neq(n-a-1, a)$ and $(k, j) \neq(n-b, b)$.

Proof. Consider the $n$-block collection

$$
\mathscr{B}=\left(E_{1}^{0}, E_{1}^{1}, \ldots, E_{1}^{n}\right)=\left({ }^{\circ} Q_{n}, \mathcal{O}_{Q_{n}}(1), \ldots, \mathcal{O}_{Q_{n}}(n-1), \Sigma(n-1)\right)
$$

of type $(1, \ldots, 1)$ of coherent sheaves on $Q_{n}$ which generate $\mathscr{D}$. According to Example 1.15 (4), its left dual $n$-block collection is

$$
\mathscr{H}=\left(R^{(0)} E_{1}^{n}, R^{(1)} E_{1}^{n-1}, \ldots, R^{(n)} E_{1}^{0}\right)=\left(\Sigma(n-1), \psi_{n-1}(n), \ldots, \psi_{0}(n)\right) .
$$

So, we have

$$
\left(R^{(0)} E_{1}^{n}\right)^{\vee}=\Sigma(n-1)^{\vee}=\Sigma(-n), \quad\left(R^{(m)} E_{1}^{n-m}\right)^{\vee}=\psi_{n-m}^{\vee}(-n), \quad 1 \leq m \leq n .
$$

Observe that the vector bundle $\left(E_{1}^{a}\right)^{\vee} \otimes E_{1}^{n}=\mathscr{O}_{Q_{n}}(a) \otimes \Sigma(n-1)=\Sigma(n-1-a)$ is generated by global sections since $n-1-a \geq 0$. Therefore, the corollary now follows from Theorem 2.4 applied to the pair $\left(E_{1}^{a}=\mathrm{O}_{Q_{n}}(a), E_{1}^{n}=\Sigma(n-1)\right)$ in (a) and the pair $\left(E_{1}^{a}=\mathrm{O}_{Q_{n}}(a), E_{1}^{b}=\mathrm{O}_{Q_{n}}(b)\right)$ in (b). 
We now state two cases on the smooth hyperquadric $Q_{n} \subset \mathbb{P}^{n+1}$ with $n \geq 2$ even.

Corollary 2.8. Let $E$ be a vector bundle on the smooth hyperquadric $Q_{n} \subset \mathbb{P}^{n+1}$ with $n \geq 2$ even. Then

(a) E is a Steiner bundle of type $\left({ }^{\circ} Q_{n}(a),{ }^{\circ} Q_{n}(b)\right)$, for some $-n+1 \leq a<b \leq 0$, given by an exact sequence

$$
0 \rightarrow \mathrm{O}_{Q_{n}}(a)^{S} \rightarrow \mathrm{O}_{Q_{n}}(b)^{t} \rightarrow E \rightarrow 0
$$

\section{if and only if}

(a1) $h^{-a-1}\left(E \otimes \psi_{-a}\right)=s$;

(a2) $h^{-b}\left(E \otimes \psi_{-b}\right)=t$;

(a3) $\quad h^{k}\left(E \otimes \psi_{j}\right)=h^{k}\left(E \otimes \Sigma_{ \pm}^{\vee}\right)=0$ for all $0 \leq k \leq n, 0 \leq j \leq n-1,(k, j) \neq$ $(-a-1,-a)$ and $(k, j) \neq(-b,-b)$.

(b) E is a Steiner bundle of type $\left(\Sigma \pm(-n),{ }^{\circ} Q_{n}(a)\right)$, for some $-n+1 \leq a \leq 0$, given by an exact sequence

$$
0 \rightarrow \Sigma \pm(-n)^{s} \rightarrow \mathrm{O}_{Q_{n}}(a)^{t} \rightarrow E \rightarrow 0
$$

\section{if and only if}

(b1) $\quad h^{n-1}\left(E \otimes \Sigma \pm^{\vee}\right)=s$;

(b2) $h^{-a}\left(E \otimes \psi_{-a}\right)=t$;

(b3) $\quad h^{k}\left(E \otimes \Sigma \pm^{\vee}\right)=0$, for $0 \leq k \leq n, k \neq n-1$, and $h^{k}\left(E \otimes \Sigma \mp^{\vee}\right)=h^{k}\left(E \otimes \psi_{j}\right)=$ 0 , for $0 \leq k \leq n, 0 \leq j \leq n-1,(k, j) \neq(-a,-a)$.

Proof. Consider the $n$-block collection

$$
\mathscr{B}=\left(E_{1}^{0}, E_{2}^{0}, E_{1}^{1}, \ldots, E_{1}^{n}\right)=\left(\Sigma_{+}(-n), \Sigma_{-}(-n), \mathcal{O}_{Q_{n}}(-n+1), \ldots, \mathcal{O}_{Q_{n}}\right)
$$

of type $(2,1, \ldots, 1)$ of coherent sheaves on $Q_{n}$ which generate $\mathscr{D}$. By Example $1.15(2)$, the left dual collection of $\mathscr{B}$ is

$$
\mathscr{H}=\left(R^{(0)} E_{1}^{n}, \ldots, R^{(n-1)} E_{1}^{1}, R^{(n)} E_{1}^{0}, R^{(n)} E_{2}^{0}\right)=\left(\mathcal{O}_{Q_{n}}, \psi_{1}^{\vee}, \ldots, \psi_{n-1}^{\vee}, \Sigma_{+}, \Sigma_{-}\right) .
$$

We then have

$$
\begin{aligned}
& \left(R^{(j)} E_{1}^{n-j}\right)^{\vee}=\psi_{j}, \text { for all } 0 \leq j \leq n-1, \\
& \left(R^{(n)} E_{1}^{0}\right)^{\vee}=\Sigma_{+}^{\vee} \text { and }\left(R^{(n)} E_{2}^{0}\right)^{\vee}=\Sigma_{-}^{\vee},
\end{aligned}
$$

and the result is a consequence of Theorem $2.4 \mathrm{E}$ applied to the pair $\left(E_{1}^{a}=\mathrm{O}_{Q_{n}}(a), E_{1}^{b}=\right.$ $\left.\mathrm{O}_{Q_{n}}(b)\right)$ in (a) and the pairs $\left(E_{1}^{0}=\Sigma_{+}(-n), E_{1}^{a}=\mathcal{O}_{Q_{n}}(a)\right)$ and $\left(E_{2}^{0}=\Sigma_{-}(-n), E_{1}^{a}=\right.$ $\left.\mathrm{O}_{Q_{n}}(a)\right)$ in (b).

As already observed, Theorem 2.4 applies to coherent sheaves on any smooth projective variety of dimension $n$ which has an $n$-block collection of coherent sheaves on $X$ generating D. So, we are led to the following open and interesting problem: 
Problem 2.9. To characterise $n$-dimensional smooth projective varieties $X$ which have an $n$-block collection of coherent sheaves on $X$ generating $\mathscr{D}$.

Acknowledgments. We would like to thank to Laura Costa for her very helpful comments during the preparation of this work. We also would like to thank to the referee for the valuable suggestions which considerably improved and shortened our paper.

\section{References}

[1] Ancona V., Ottaviani G.: Unstable hyperplanes for Steiner bundles and multidimensional matrices. Adv. Geom. 1 (2001), 165-192

[2] Arrondo E., Costa L.: Vector bundles on Fano 3-folds without intermediate cohomology. Comm. Algebra 28 (2000), 3899-3911

[3] Arrondo E., Graña B.: Vector bundles on $G(1,4)$ without intermediate cohomology. J. Algebra 214 (1999), 128-142

[4] Beilinson A. A.: Coherent sheaves on $\mathbb{P}^{n}$ and problems of linear algebra. Funct. Anal. Appl. 12 (1978), 214-216

[5] Bohnhorst G., Spindler H.: The stability of certain vector bundles on $\mathbb{P}^{n}$, Complex algebraic varieties (Bayreuth, 1990). Lecture Notes in Math. 1507, pp. 39-50. Springer, Berlin 1992

[6] Brambilla C.: Cokernel bundles and Fibonacci bundles. Math. Nachr. 281 (2008), 499-516

[7] Brambilla C.: Simplicity of vector bundles on $\mathbb{P}^{n}$ and exceptional vector bundles. Ph.D. thesis, University of Florence, 2004

[8] Brambilla C., Faenzi D.: Vector bundles on Fano threefolds of genus 7 and Brill-Noether loci. Preprint (2007)

[9] Cascini P.: On the moduli space of the Schwarzenberger bundles. Pacific J. Math. 205 (2002), $311-323$

[10] Chang M.-C.: Filtered Bertini-type theorem. J. Reine Angew. Math. 397 (1989), 214-219

[11] Chiantini L., Madonna C.: ACM bundles on a general quintic threefold. Mathematiche (Catania) 55 (2000), 239-258 (2002). Dedicated to Silvio Greco on the occasion of his 60th birthday (Catania, 2001)

[12] Chiantini L., Madonna C.: A splitting criterion for rank 2 bundles on a general sextic threefold. Internat. J. Math. 15 (2004), 341-359

[13] Costa L., Miró-Roig R. M.: Cohomological characterization of vector bundles on multiprojective spaces. J. Algebra 294 (2005), 73-96

[14] Costa L., Miró-Roig R. M.: Geometric collections and Castelnuovo-Mumford regularity. Math. Proc. Cambridge Philos. Soc. 143 (2007), 557-578

[15] Costa L., Miró-Roig R. M.: $m$-Blocks collections and Castelnuovo-Mumford regularity in multiprojective spaces. Nagoya Math. J. 186 (2007), 119-155

[16] Dolgachev I., Kapranov M.: Arrangements of hyperplanes and vector bundles on $\mathbb{P}^{n}$. Duke Math. J. 71 (1993), 633-664

[17] Ein L.: Normal sheaves of linear systems on curves, Algebraic Geometry: Sundance 1988. Contemp. Math 116, pp. 9-18. Amer. Math. Soc., Providence, RI 1991

[18] Faenzi D.: Bundles over the Fano threefold $V_{5}$. Comm. Algebra 33 (2005), 3061-3080

[19] Faenzi D.: Bundles over Fano threefolds of type $V_{22}$. Ann. Mat. Pura Appl. (4) 186 (2007), 1-24

[20] Gorodentsev A. L.: Exceptional bundles on surfaces with a moving anticanonical class. Math. URSS-Izv. 33 (1989), 67-83 
[21] Gorodentsev A. L., Kuleshov S. A.: Helix Theory. Mosc. Math. J. 4 (2004), 377-440, 535

[22] Hille L.: Consistent algebras and special tilting sequences. Math. Z. 220 (1995), 189-205

[23] Horrocks G.: Vector bundles on the punctured spectrum of a local ring. Proc. London Math. Soc. (3) 14 (1964), 689-713

[24] Horrocks G.: Construction of bundles on $\mathbb{P}^{n}$. Astérisque 71-72, pp. 197-203. Société Mathématique de France, Paris, 1980, Séminaire E. N. S., 1977-1978

[25] Jardim M., Martins R. V.: Linear and Steiner bundles on projective varieties. Preprint (2007)

[26] Kapranov M.: On the derived category of coherent sheaves on Grassmann manifolds. Math. USSR-Izv. 24 (1985), 183-192

[27] Kapranov M.: On the derived category of coherent sheaves on some homogeneous spaces. Invent. Math. 92 (1988), 479-508

[28] Karpov B., Nogin D. Yu.: Three-block exceptional collections over Del Pezzo surfaces. Izv. Math. 62 (1998), 429-463

[29] Madonna C.: A splitting criterion for rank 2 vector bundles on hypersurfaces in $\mathbb{P}^{4}$. Rend. Sem. Mat. Univ. Politec. Torino 56 (1998), 43-54 (2000).

[30] Madonna C.: Rank-two vector bundles on general quartic hypersurfaces in $\mathbb{P}^{4}$. Rev. Mat. Complut. 13 (2000), 287-301

[31] Madonna C.: Rank 4 vector bundles on the quintic threefold. Cent. Eur. J. Math. 3 (2005), 404-411 (electronic)

[32] Miró-Roig R. M.: A cohomological class of vector bundles. Proc. Amer. Math. Soc. 120 (1994), $1003-1008$

[33] Ottaviani G.: Critères de scindage pour les fibrés vectoriels sur les Grassmanniennes et les quadriques. C. R. Acad. Sci. Paris Sér. I Math. 305 (1987), 257-260

[34] Ottaviani G.: Spinor bundles on quadrics. Trans. Amer. Math. Soc. 307 (1988), 301-316

[35] Ottaviani G.: Some extensions of Horrocks criterion to vector bundles on Grassmannians and quadrics. Ann. Mat. Pura Appl. (4) 155 (1989), 317-341

[36] Soares H.: Steiner bundles on the hyperquadric $Q_{n} \subset \mathbb{P}^{n+1}, n \geq 3$. Comm. Algebra 35 (2007), 4116-4136

[37] Soares H.: Steiner vector bundles on algebraic varieties. Ph.D. thesis, University of Barcelona 2008

[38] Vallès J.: Fibrés de Schwarzenberger et coniques de droites sauteuses. Bull. Soc. Math. France 128 (2000), 433-449

[39] Vallès J.: Nombres maximal d'hyperplans instables pour un fibré de Steiner. Math. Z. 233 (2000), $507-514$

Received December 5, 2007; in final form March 21, 2008

Facultat de Matemàtiques, Departament d'Àlgebra i Geometria, Universitat de Barcelona, Gran Via de les Corts Catalanes 585, 08007 Barcelona, Spain

miro@ub.edu

Departamento de Métodos Quantitativos, ISCTE, Av. das Forças Armadas, 1649-026 Lisboa, Portugal helena.soares@iscte.pt 
Copyright of Forum Mathematicum is the property of Walter de Gruyter GmbH \& Co. KG. and its content may not be copied or emailed to multiple sites or posted to a listserv without the copyright holder's express written permission. However, users may print, download, or email articles for individual use. 